\title{
Presentación
}

\author{
Steffen Bay Rasmussen \\ Profesor de Relaciones Internacionales, Universidad de Deusto \\ steffen.bayrasmussen@deusto.es
}

doi: http://dx.doi.org/10.18543/ced-64-2021pp23-26

Resumen: El proceso de integración europea ha evolucionado a través de crisis de gobernanza hacia una integración cada vez mayor de las sociedades europeas, provocando nuevas preguntas sobre la organización política del continente europeo. A la vez, las sociedades europeas se han hecho más diversas, haciendo surgir nuevos y complejos problemas de coexistencia. Ahora, Europa también debe gestionar las consecuencias de su modelo económico basado en el consumo de recursos finitos. Más allá de crisis y acontecimientos específicos, Europa se enfrenta, por tanto, a un desafío multifacético de sostenibilidad ecológica, democrática y social. Abordar los desafíos desde el punto de vista de la sostenibilidad significa ver la viabilidad ecológica, democrática y social a largo plazo de Europa como posible gracias a la reconstrucción continua de las sociedades europeas mediante prácticas culturales, sociales, económicas y políticas innovadoras, dentro de las limitaciones ecológicas definidas por los límites de nuestro planeta.

Palabras clave: Sostenibilidad, democracia, diversidad, confianza social, conceptos mágicos, Spitzenkandidaten, desarrollo sostenible, democracia participativa; Iniciativa Ciudadana Europea, turismo comunitario, imaginario, percepción de amenazas, encuesta factorial

Europa ha sido descrita como un modelo que está continuamente «fallando hacia adelante» a través de crisis hacia una integración institucional cada vez mayor, respaldada por un aumento de la eficiencia económica y de la prosperidad. Sin embargo, el cambio climático ha dejado perfectamente claro que el modelo actual de expansión económica basado en el consumo de recursos finitos y la contaminación continua del medio ambiente natural no puede seguir formando la base del sustento material de la integración social en Europa.

Al mismo tiempo, también estamos siendo testigos de cómo la discusión sobre la finalité o «punto final» de la integración europea está cada vez más en la agenda política, con partidos políticos y movimientos sociales que representan partes sustanciales de la población europea cuestionando abiertamente la situación actual en Europa, así como las lógicas de desbor- 
damiento funcional en la integración europea asociado con el enfoque de «fallar hacia adelante», y abogando abiertamente por un spill-back, que implica devolver competencias y funciones al estado y reconstruyendo el estado-nación como el lugar más importante para las orientaciones identitarias y la solidaridad política y social.

Además, estas agendas a menudo incluyen una securitización de la diferencia y una fuerte dicotomía yo-otro, construyendo la diversidad y la movilidad humana como una amenaza por motivos económicos, culturales, sociales y religiosos. De hecho, la noción del Antropoceno enfatiza precisamente la interconexión de los problemas y luchas ecológicas, sociales, económicas y políticas, ejemplificada por las guerras por los recursos naturales, el desafío de gestionar una economía circular y el cambio climático que a su vez da lugar a una mayor movilidad humana, desafíos para la redistribución de la riqueza en las sociedades y el agravamiento de las divisiones sociales y políticas existentes y la creación de nuevas.

Mientras que el triple desafío ecológico, democrático y social que enfrenta Europa puede conceptualizarse en términos de búsqueda de soluciones a las crisis, un enfoque desde el concepto de sostenibilidad mantiene una visión más amplia de cualquier problema específico con una conciencia de los patrones más amplios de cambio ecológico, político y social a principios del siglo XXI, en Europa y más allá. En general, se ha pensado en la sostenibilidad en términos de crear un equilibrio entre las necesidades y aspiraciones humanas actuales y futuras en la intersección de la macroeconomía (crecimiento del PIB), el medio ambiente (ecosistemas equilibrados) y el campo social (educación, empleo), que a su vez ha generado críticas al concepto por tener un sesgo analítico y político inherentemente conservador.

Sin embargo, la noción de una Europa sostenible no tiene por qué implicar una Europa estática, reaccionaria y autosuficiente que mira hacia adentro. Al contrario, también podría conceptualizarse como un concepto dinámico que considera que la viabilidad ecológica, democrática y social a largo plazo es posible gracias a la reconstrucción continua de las sociedades europeas mediante prácticas culturales, sociales, económicas y políticas innovadoras, dentro de las limitaciones ecológicas definidas por los límites de nuestro planeta.

Es en este contexto que se ha creado el número 64/2021 de los Cuadernos Europeos de Deusto que incluye 4 artículos sobre la sostenibilidad en y de Europa. Fueron redactados como parte del Programa Intensivo del Máster Erasmus Mundus en Eurocultura organizado por la Universidad de Deusto en junio de 2020 bajo el título «¿Una Europa sostenible? Sociedad, política y cultura en el Antropoceno» y en el que participaron más de 100 estudiantes académicos del Consorcio Eurocultura, que además de la Uni- 
versidad de Deusto incluye: Universidad de Groningen, Georg-AugustUniversität Göttingen, Universidad Jagelloniana de Cracovia, Universidad Palacký de Olomouc, Universidad de Estrasburgo, Universidad de Udine, Universidad de Uppsala.

Trond Ove Tøllefsen abre esta edición temática con una examinación sistemática y crítica del concepto de sostenibilidad y sus propiedades mágicas, entendido como su amplitud de significado, connotaciones positivas, adopción consensuada y uso extendido. A través de un análisis horizontal y vertical, el artículo cuestiona el significado de sostenibilidad en el contexto de la historial larga del impacto humano en la naturaleza. Concluye que las propiedades mágicas del concepto de sostenibilidad han permitido generar un discurso compartido sobre el uso responsable de recursos naturales que moviliza a la acción, a pesar de que estas mismas propiedades mágicas también ha posibilitado su mal uso estratégico.

Carlos Espaliú Berdud, pone el foco a la sostenibilidad democrática en la UE, concretamente en la parlamentarización del sistema político y las experiencias con los nombramientos del presidente de la Comisión en 2014 y 2019. Mientras que el nombramiento de 2014 reflejó un funcionamiento del sistema Spitzenkandidaten que podría considerarse un fortalecimiento de la parlamentarización del sistema político de la UE, el nombramiento de 2019 mostró un resultado opuesto. El autor concluye que esto fue el resultado de una falta de respeto por los resultados de las elecciones parlamentarias europeas que contribuyó a disminuir la legitimidad de la UE y, en última instancia, disminuir la sostenibilidad democrática de la UE como sistema político entre la organización internacional y el estado federal.

Edurne Bartolomé, Hermann Dülmer y Lluis Coromina se centran en la sostenibilidad social de Europa, concretamente en cómo la diversidad en la sociedad genera percepciones de amenaza o confianza entre diferentes grupos. La base empírica del artículo es una encuesta factorial realizada en Bilbao y Colonia que examina el impacto en la confianza social de diferentes factores como la edad, el color de piel, la religión y las condiciones socioeconómicas. Los autores concluyen que los factores socioeconómicos son más importantes que los culturales en términos de confianza social, pero también que quedan numerosos obstáculos para la consolidación de la confianza social en el actual contexto de diversidad. La creación de una identidad europea más integradora y una Europa socialmente sostenible requerirá, por tanto, un cambio en las actitudes generales, a través de la educación y el relevo generacional.

Monica Pascoli analiza el impacto del turismo en la sostenibilidad, con especial énfasis en la sostenibilidad social de las comunidades locales. La autora analiza cómo un imaginario turístico surge como resultado de la negociación de diferentes actores. El artículo está basado en entrevistas reali- 
zadas en Paularo, Italia. En este caso, no se encontraron construcciones disonantes entre los actores, aunque sí se encontró una gran diferencia en la interpretación del patrimonio natural y cultural entre locales y profesionales del turismo, tanto en términos de cómo debe ser vivido como del significado que se atribuye a los diferentes sitios. Esto indica además un gran potencial para los enfoques comunitarios del turismo como facilitadores de la sostenibilidad social.

\section{Sobre el autor}

Steffen Bay Rasmussen es profesor de Relaciones Internacionales en la Universidad de Deusto y Director del Máster Erasmus Mundus en Eurocultura: Sociedad, política y cultura en un contexto global. Imparte cursos de Derecho Internacional Público y Relaciones Internacionales, incluyendo historia y teoría de la integración europea, integración regional comparada y diplomacia pública y ha sido profesor invitado e investigador en varias universidades, como el Instituto Holandés de Relaciones Internacionales (Clingendael), la Universidad Sultan Qaboos (Omán), la Universidad de Navarra (España) y la Universidad del País Vasco (España). Su investigación actual se centra en la estrategia internacional de la UE y las relaciones diplomáticas, la diplomacia pública y la identidad internacional de la UE y la seguridad ontológica. Sus publicaciones recientes incluyen «The ideas and practices of the European Union's structural antidiplomacy: An unstable equilibrium», Boston, Brill. 


\section{Derechos de autor}

Los derechos de autor (para la distribución, comunicación pública, reproducción e inclusión en bases de datos de indexación y repositorios institucionales) de esta publicación (Cuadernos Europeos de Deusto, CED) pertenecen a la editorial Universidad de Deusto. El acceso al contenido digital de cualquier número de Cuadernos Europeos de Deusto es gratuito inmediatamente después de su publicación. Los trabajos podrán leerse, descargarse, copiar y difundir en cualquier medio sin fines comerciales y según lo previsto por la ley; sin la previa autorización de la Editorial (Universidad de Deusto) o el autor. Así mismo, los trabajos editados en CED pueden ser publicados con posterioridad en otros medios o revistas, siempre que el autor indique con claridad y en la primera nota a pie de página que el trabajo se publicó por primera vez en CED, con indicación del número, año, páginas y DOI (si procede). Cualquier otro uso de su contenido en cualquier medio o formato, ahora conocido o desarrollado en el futuro, requiere el permiso previo por escrito del titular de los derechos de autor.

\section{Copyright}

Copyright (for distribution, public communication, reproduction and inclusion in indexation databases and institutional repositories) of this publication (Cuadernos Europeos de Deusto, CED) belongs to the publisher University of Deusto. Access to the digital content of any Issue of Cuadernos Europeos de Deusto is free upon its publication. The content can be read, downloaded, copied, and distributed freely in any medium only for non-commercial purposes and in accordance with any applicable copyright legislation, without prior permission from the copyright holder (University of Deusto) or the author. Thus, the content of CED can be subsequently published in other media or journals, as long as the author clearly indicates in the first footnote that the work was published in CED for the first time, indicating the Issue number, year, pages, and DOI (if applicable). Any other use of its content in any medium or format, now known or developed in the future, requires prior written permission of the copyright holder. 